\title{
AMENABILITY AND SEMISIMPLICITY FOR SECOND DUALS OF QUOTIENTS OF THE FOURIER ALGEBRA $A(G)$
}

\author{
EDMOND E. GRANIRER
}

(Received 23 April 1996; revised 8 April 1997)

\author{
Communicated by G. Robertson
}

\begin{abstract}
Let $F \subset G$ be closed and $A(F)=A(G) / I_{F}$. If $F$ is a Helson set then $A(F)^{* *}$ is an amenabie (semisimple) Banach algebra. Our main result implies the following theorem: Let $G$ be a locally compact group, $F \subset G$ closed, $a \in G$. Assume either (a) For some non-discrete closed subgroup $H$, the interior of $F \cap a H$ in $a H$ is non-empty, or (b) $R \subset G, S \subset R$ is a symmetric set and $a S \subset F$. Then $A(F)^{* *}$ is a non-amenable non-semisimple Banach algebra. This raises the question: How 'thin' can $F$ be for $A(F)^{* *}$ to remain a non-amenable Banach algebra?
\end{abstract}

1991 Mathematics subject classification (Amer. Math. Soc.): primary 46H20, 43A30; secondary 43A20.

\section{Introduction}

Let $G$ be a locally compact group and $J \subset A(G)$ a closed ideal with zero set $Z(J)=\{x \in G ; u(x)=0$ for all $u \in J\}=F$. Consider the second dual $(A(G) / J)^{* *}$, of the quotient algebra $A(G) / J$, equipped with Arens multiplication.

If $F \subset G$ is a Helson set (thus $A(F)=C(F)$ ) then $A(F)^{* *}$ is a commutative $C^{*}$ algebra and is hence amenable, by a result of Sheinberg (see [CL] for amenability of Banack. algebras and references).

The subset $S$ of the real line $R$ is symmetric if there are $t_{n}>0$ such that $t_{n}>\sum_{n+1}^{\infty} t_{i}$ for all $n \geq 1$ and $S=\left\{\sum_{0}^{\infty} \varepsilon_{i} t_{i} ; \varepsilon_{i}=0,1\right\}$. The Cantor $1 / 3$ set is such (see [GMc, p. 88]). Analogously for $S \subset T$ (the unit circle). This is the only sense in which 'symmetric' is used in this paper.

Our main result implies the

THEOREM. Let $G$ be a locally compact group $J \subset A(G)$ a closed ideal with (C) 1997 Australian Mathematical Society 0263-6115/97 \$A2.00+0.00 
$Z(J)=F$ and $a, b \in G$. Assume one of

(a) For some non-discrete closed subgroup of $H \subset G$, int ${ }_{a H b} F \neq \emptyset$ or

(b) $R$ is a closed subgroup of $G, S \subset R$ is a symmetric set with a $b \subset F$.

Then $(A(G) / J)^{* *}$ is a non-amenable non-semisimple Banach algebra.

Furthermore, in case (a) the result holds for the algebras $A_{p}(G) / J, 1<p<\infty$, where $A_{2}(G)=A(G)$ is the Fourier algebra of $G$; see $[\mathrm{Hz} 1]$.

As a very mild consequence one gets that $A(G)^{* *}$ (or $B(G)^{* *}$ ) is an amenable Banach algebra if and only if $G$ is finite. This is a dual result of a theorem of Ghahramani, Loy and Willis [GLW] who have shown that $L^{1}(G)^{* *}$ (or $\left.M(G)^{* *}\right)$ is amenable if and only if $G$ is finite. And yet, if $E \subset Z$ is a Sidon set $A(E)^{* *}$ is amenable (see the sequel).

\section{Notation and definitions}

We follow the notation in [Ey] for the Fourier algebra $A(G)$, except that we denote its dual module $A(G)^{*}$, where $(u \cdot \Phi, v)=(\Phi, u v)$ for $u, v \in A(G), \Phi \in A(G)^{*}$, by $P M(G)$, while $V N(G)$ is used in [Ey]. Thus $B(G)$ is the linear span of the continuous positive definite functions on $G$.

If $\mu \in M(G)$, the bounded Borel measures on $G$, let $\lambda \mu \in P M(G)$ be given by $(\lambda \mu, v)=\int v d \mu$ where $v \in A(G)$. Thus $\left(\lambda \delta_{x}, v\right)=v(x)$ if $x \in G, v \in A(G)$. If $\Phi \in P M(G)$, denote its support by supp $\Phi$. Thus $x \in \operatorname{supp} \Phi$ if and only if for each neighborhood $V$ of $x$ there is some $v \in A(G)$ such that supp $v \subset V$ and $(\lambda \mu, v) \neq 0$.

If $\boldsymbol{P} \subset P M(G)$ is a $w^{*}$ closed $A(G)$ module and $a \in G$, let $E_{\mathbf{P}}(a)=\operatorname{ncl}\{\Phi \epsilon$ $\boldsymbol{P}: a \notin \operatorname{supp} \Phi\}$ (where ncl denotes norm-closure), $\operatorname{TI}_{\mathbf{P}}(a)=\left\{\Psi \in \boldsymbol{P}^{*}: \Psi=0\right.$ on $\left.E_{\mathrm{P}}(a)\right\}$ and, if $\lambda \delta_{a} \in \boldsymbol{P}$, $\operatorname{TIM}_{\mathbf{P}}(a)=\left\{\Psi \in \boldsymbol{P}^{*}:\left(\Psi, \lambda \delta_{a}\right)=1=\|\Psi\|, \Psi=0\right.$ on $\left.E_{\mathrm{P}}(a)\right\}$ (this being the set of topologically invariant means on $\boldsymbol{P}$ at $a$ ).

Let $J \subset A(G)$ be a closed ideal with zero set $Z(J)=F$. Let $P=(A(G) / J)^{*}$ where $A(G) / J$ is taken with the quotient norm. If $F \subset G$ is closed then $I_{F}=\{v \in$ $A(G): v=0$ on $F\}$ and $A(F)=A(G) / I_{F}$. We consider $\boldsymbol{P}^{*}=(A(G) / J)^{* *}$ equipped with the Arens multiplication given by $\left(\Psi_{1} \square \Psi_{2}, \Phi\right)=\left(\Psi_{1}, \Psi_{2} \square \Phi\right)$ for $\Psi_{1}, \Psi_{2} \in P^{*}$, $\Phi \in P$ where $\Psi_{2} \square \Phi \in P$ is given by $\left(\Psi_{2} \square \Phi, u\right)=\left(\Psi_{2}, u \cdot \Phi\right)$ for $u \in A(G) / J$; see $[\mathrm{DH}]$. Thus $\left(\boldsymbol{P}^{*}, \square\right)$ is a Banach algebra in which $A(G) / J$ is embedded and $\square$ extends the multiplication in $A(G) / J$.

The Banach algebras $A_{p}(G), 1<p<\infty$, are as defined in [Hzl] and are such that $A_{2}(G)=A(G)$ is the Fourier algebra of $G$. Thus $P M_{p}(G)=A_{p}(G)^{*}$. Let $B_{p}^{M}(G)=B_{p}^{M}=\left\{v \in C(G): v u \in A_{p}\right.$ for all $\left.u \in A_{p}\right\}$, where $C(G)\left[C_{c}(G)\right]$ are the bounded continuous [with compact support] functions on $G$. The reader not interested in these may assume that $A_{p}(G)$ is $A(G)$ and proceed. 
In any case, as in [Gr2, Gr3], the above notation makes sense for $\boldsymbol{P} \subset \boldsymbol{P} M_{p}(G)$. If $p \neq 2$ then $A_{p}(G)$ is very different from $A(G)$; see [Gr2, p. 49].

We denote for simplicity $A(G)$ by $A, A_{p}(G)$ by $A_{p}$, and $A_{p}(F)=A_{p} / I_{F}$.

If $F, H$ are subsets of $G$ then int ${ }_{H} F$ is the interior of $F \cap H$ in $H$ (with the relative topology from $G)$. If $u \in C(G)$, let $\operatorname{supp} u=\operatorname{cl}\{x: u(x) \neq 0\}$.

\section{The main results}

In what follows, let $J \subset A_{p}$ be a closed ideal with $F=Z(J)$ and $\boldsymbol{P}=\left(A_{p} / J\right)^{*}$.

LEMMA 1. (a) $\Psi \in \operatorname{TI}_{\mathbf{P}}(a)$ if and only if $(*)(\Psi, u \cdot \Phi)=u(a)(\Psi, \Phi)$ for all $u \in B_{p}^{M}$ and $\Phi \in \boldsymbol{P}$. (b) $\operatorname{TIM}_{\mathbf{P}}(a) \neq \emptyset$ for all $a \in F$.

PROOF. (a) The proof of Lemma $8^{\prime}$ of [Gr3] holds for all locally compact groups $G$ with $A(G), B(G)$ replaced by $A_{p}(G), B_{p}^{M}(G)$, since only results in [Hzl] (which are valid in this context) were used in its proof. Thus $E_{\mathbf{P}}(a)=\operatorname{ncl}\{\Phi-v \cdot \Phi: \Phi \in \boldsymbol{P}$, $\left.v \in S_{3}(a)\right\}$ where $S_{3}(a)=\left\{v \in B_{p}^{M}: v(a)=1\right\}$. Thus $\Psi \in \operatorname{TI}_{\mathbf{P}}(a)$ if and only if $(\Psi, u \cdot \Phi)=(\Psi, \Phi)$ for all $\Phi \in P$ and $u \in S_{3}(a)$. If $\Psi \in \operatorname{TI}_{\mathbf{P}}(a)$ and $u \in B_{p}^{M}$ with $u(a) \neq 0$, then $(\Psi, u \cdot \Phi)=u(a)(\Psi, \Phi)$ for all $\Phi \in P$. If now $u \in B_{p}^{M}$ and $u(a)=0$, then since $1 \in B_{p}^{M}$ we have $(\Psi,(1-u) \cdot \Phi)=(1-u(a))(\Psi, \Phi)=(\Psi, \Phi)$. Thus $(\Psi, u \cdot \Phi)=0$ and $(*)$ holds for $\Psi$. If now $\Psi \in P^{*}$ and $(*)$ holds for $\Psi$ then $(\Psi, \Phi-v \cdot \Phi)=0$ if $\Phi \in \boldsymbol{P}$ and $v \in S_{3}(a)$. Thus $\Psi=0$ on $E_{\mathbf{P}}(a)$.

(b) This is shown for example as in [Gr2, p. 122] with $e$ replaced by $a \in F$.

LEMMA 2. (a) If $a \in F$ then $\Psi \rightarrow\left(\Psi, \lambda \delta_{a}\right)$ is a multiplicative $w^{*}$-continuous non-zero linear functional on $\boldsymbol{P}^{*}$.

(b) If $\Psi \in P^{*}, \Psi_{1} \in \operatorname{TI}_{\mathbf{P}}(a)$ then $\Psi \square \Psi_{1}=\left(\Psi, \lambda \delta_{a}\right) \Psi_{1}$.

(c) If $I_{a}=\left\{\Psi \in \boldsymbol{P}^{*}:\left(\Psi, \lambda \delta_{a}\right)=0\right\}$ then $I_{a} \square \operatorname{TI}_{\mathbf{P}}(a)=\{0\}$.

(d) For any $u \in A_{p} / J, \Phi \in P, \Psi \in P^{*}, \Psi \square(u \cdot \Phi)=u \cdot(\Psi \square \Phi)$.

PROOF. (a) holds by [DH, p. 316]. Alternatively, note that $\Psi \square \lambda \delta_{a}=\left(\Psi, \lambda \delta_{a}\right) \lambda \delta_{a}$.

(b) If $u \in A_{p}, \Phi \in \boldsymbol{P}$ then $\left(\Psi_{1} \square \Phi, u\right)=\left(\Psi_{1}, u \cdot \Phi\right)=u(a)\left(\Psi_{1}, \Phi\right)=$ $\left(\left(\Psi_{1}, \Phi\right) \lambda \delta_{a}, u\right)$. Hence $\left(\Psi \square \Psi_{1}, \Phi\right)=\left(\Psi, \Psi_{1}(\Phi) \lambda \delta_{a}\right)=\left(\left(\Psi, \lambda \delta_{a}\right) \Psi_{1}, \Phi\right)$.

(c) Immediate from (b).

(d) If $v \in A_{p} / J$ then $(\Psi \square(u \cdot \Phi), v)=(\Psi,(u v) \cdot \Phi)=(\Psi \square \Phi, u v)=$ $(u \cdot(\Psi \square \Phi) v)$.

PROPOSITION 3. For any $a, b \in F, a \neq b$ :

(a) $\operatorname{TI}_{\mathbf{P}}(a)$ is a non-zero $w^{*}$-closed two-sided ideal of $\left(\boldsymbol{P}^{*}, \square\right)$ such that $I_{a} \square \operatorname{TI}_{\mathbf{P}}(a)=$ $\{0\}$. 
(b) $\operatorname{TI}_{\mathbf{P}}^{0}(a)=I_{a} \cap \operatorname{TI}_{\mathbf{P}}(a)$ is a w*-closed two-sided ideal such that $\operatorname{TI}_{\mathbf{P}}^{0}(a) \square \operatorname{TI}_{\mathbf{P}}^{0}(a)=$ $\{0\}$.

If card $\operatorname{TIM}_{P}(a) \geq 2$ then $\operatorname{TI}_{\mathbf{P}}^{0}(a) \neq\{0\}$.

(c) $\operatorname{TI}_{\mathbf{P}}(a) \cap \mathrm{TI}_{\mathbf{P}}(b)=\{0\}$.

ProOf. (a) $\operatorname{TI}_{\mathbf{P}}(a)$ is a left ideal by Lemma 2(b). If $\Psi_{1} \in \operatorname{TI}_{\mathbf{P}}(a), \Psi \in \boldsymbol{P}^{*}$, $u \in A_{p} / J$, and $\Phi \in P$, then $\left(\Psi_{1} \square \Psi, u \cdot \Phi\right)=\left(\Psi_{1}, \Psi \square(u \cdot \Phi)\right)=\left(\Psi_{1}, u \cdot(\Psi \square \Phi)\right)$ (by Lemma $2(\mathrm{~d}))=u(a)\left(\Psi_{1} \square \Psi, \Phi\right)$ (by Lemma 1). Hence, again by Lemma 1 , $\Psi_{1} \square \Psi \in \operatorname{TI}_{\mathbf{P}}(a)$. Now $\Psi \square \Psi_{1}=\left(\Psi, \lambda \delta_{a}\right) \Psi_{1}$ by Lemma 2(b). Thus $I_{a} \square \operatorname{TI}_{\mathbf{P}}(a)=0$.

(b) Since $I_{a}$ and $\operatorname{TI}_{\mathbf{P}}(a)$ are $w^{*}$-closed two-sided ideals, $\operatorname{TI}_{\mathbf{P}}^{0}(a)$ is such and even $I_{a} \square \operatorname{TI}_{\mathbf{P}}(a)=\{0\}$. If $\Psi_{1} \neq \Psi_{2}$ are in $\operatorname{TIM}_{\mathbf{P}}(a)$ then $0 \neq \Psi_{1}-\Psi_{2} \in I_{a} \cap \operatorname{TI}_{\mathbf{P}}(a)=$ $\operatorname{TI}_{\mathbf{P}}^{0}(a)$.

(c) If $\Psi \in \operatorname{TI}_{\mathbf{P}}(a) \cap \operatorname{TI}_{\mathbf{P}}(b)$ then $(\Psi, u \cdot \Phi)=u(a)(\Psi, \Phi)=u(b)(\Psi, \Phi)$ for $u \in A_{p} / J, \Phi \in P$. If we choose $u \in A_{p} / J$ with $u(a)=0, u(b) \neq 0$, we get $(\Psi, \Phi)=0$; thus $\Psi=0$.

THEOREM 4. (a) If $\Psi_{0} \in \operatorname{TIM}_{\mathbf{P}}(a)$ then $\Psi \rightarrow \Psi_{0} \square \Psi$ is a projection operator from $\boldsymbol{P}^{*}$ onto the two-sided ideal $\operatorname{TI}_{\mathbf{P}}(a)$. Thus $\boldsymbol{P}^{*}=\operatorname{TI}_{\mathbf{P}}(a) \oplus\left\{\Psi-\Psi_{0} \square \Psi: \Psi \in \boldsymbol{P}^{*}\right\}$.

(b) If card $\operatorname{TIM}_{\mathbf{P}}(a) \geq 2$ then $\operatorname{TI}_{\mathbf{P}}(a)$ has no (even unbounded) right approximate identity, and is hence a non-amenable $w^{*}$-closed ideal of $\boldsymbol{P}^{*}$.

(c) If card $\operatorname{TIM}_{\mathrm{P}}(a) \geq 2$ for some $a \in F$ then $\boldsymbol{P}^{*}$ is a non-amenable non-semisimple Banach algebra.

PROOF. (a) Let $Q(\Psi)=\Psi_{0} \square \Psi$. If $\Psi \in \operatorname{TI}_{\mathbf{P}}(a)$ then $Q(\Psi)=\left(\Psi_{0}, \lambda \delta_{a}\right) \Psi=\Psi$ by Lemma 2(b). For any $\Psi \in P^{*}, Q^{2}(\Psi)=\Psi_{0} \square\left(\Psi_{0} \square \Psi\right)=\Psi_{0} \square \Psi=Q(\Psi)$ since $\Psi_{0} \square \Psi \in \operatorname{TI}_{\mathbf{P}}(a)$ by Proposition 3(a).

If now $Q \Psi=\Psi$ then $\Psi_{0} \square \Psi=\Psi$ hence $\Psi \in \operatorname{TI}_{P}(a)$ by Proposition 3(a). Thus $P^{*}=Q P^{*} \oplus(I-Q) P^{*}=\operatorname{TI}_{\mathbf{P}}(a) \oplus\left\{\Psi-\Psi_{0} \square \Psi: \Psi \in \boldsymbol{P}^{*}\right\}$ where $I: P^{*} \rightarrow P^{*}$ is the identity.

(b) Let $\Psi_{\alpha} \subset \operatorname{TI}_{\mathbf{P}}(a)$ be a right approximate identity. Let $\Psi_{1} \neq \Psi_{2}$ be in $\operatorname{TIM}_{\mathbf{P}}(a)$. Thus $\Psi_{1}-\Psi_{2} \in I_{a}$. Hence, by Proposition 3(a), $\left(\Psi_{1}-\Psi_{2}\right) \square \Psi_{\alpha}=0$. But $\Psi_{1} \leftarrow$ $\Psi_{1} \square \Psi_{\alpha}=\Psi_{2} \square \Psi_{\alpha} \rightarrow \Psi_{2}$ which cannot be.

(c) If $\Psi_{1} \neq \Psi_{2}$ are in $\operatorname{TIM}_{\mathbf{P}}(a)$ then $0 \neq \Psi_{1}-\Psi_{2} \in \operatorname{TI}_{\mathbf{P}}^{0}(a)$ and $\operatorname{TI}_{\mathbf{P}}^{0}(a) \square \operatorname{TI}_{\mathbf{P}}^{0}(a)=$ $\{0\}$. Hence $\{0\} \neq \operatorname{TI}_{\mathbf{P}}^{0}(a) \subset \operatorname{rad} \boldsymbol{P}^{*}$ and $\boldsymbol{P}^{*}$ is not semisimple.

If now $\boldsymbol{P}^{*}$ is an amenable Banach algebra then $\operatorname{TI}_{\mathbf{P}}(a)$ is a $w^{*}$ - (hence norm-) closed two-sided ideal which is complemented in $\boldsymbol{P}^{*}$; hence $\mathrm{TI}_{\mathbf{P}}(a)^{\perp}$ is complemented in $\boldsymbol{P}^{* *}$. But then by Khelemskii's Theorem (see [CL, p. 97, Thm 3.7]) $\mathrm{TI}_{\mathbf{P}}(a)$ has a bounded approximate identity, which cannot be the case by (b).

REMARK. Note that if $\Psi_{0} \in \operatorname{TIM}_{\mathbf{P}}(a)$ then $\left\{\Psi-\Psi_{0}: \Psi \in \operatorname{TIM}_{\mathbf{P}}(a)\right\} \subset \operatorname{TI}_{\mathbf{P}}^{0}(a) \subset$ $\operatorname{rad} \boldsymbol{P}^{*}$. 
We recall now some results of ours in the next theorem. The set $D_{1}(J) \subset F$ was defined in [Gr3] by: $a \in D_{1}(J)$ if there exists a sequence $u_{n} \in A_{p}$ with compact supports such that (i) $1=u_{n}(a)=\left\|u_{n}\right\|$, (ii) $\left\{F \cap \operatorname{supp} u_{n}\right\}$ is a neighborhood base in $F$ at $a$, and (iii) there is some $d>0$ such that $\left\|\sum_{1}^{n} \alpha_{k} u_{k}^{\prime}\right\| \geq d \sum_{1}^{n}\left|\alpha_{k}\right|$, for all $n$ and $\alpha_{k} \in C$, where $u_{k}^{\prime}=u_{k}+J \in A_{p} / J$. Note that (iii) can be replaced by: (iii) $\left\{u_{k}^{\prime}\right\}$ has no weak Cauchy subsequence (by Rosenthal's Theorem).

THEOREM 5. Let $G$ be a locally compact group, $J$ a closed ideal of $A_{p}(G)$, $1<p<\infty$, with $Z(J)=F, a, b \in G$ and $\boldsymbol{P}=\left(A_{p}(G) / J\right)^{*}$.

(i) If $D_{1}(J) \neq \emptyset$ and $x \in D_{1}(J)$ then card $\operatorname{TIM}_{\mathbf{P}}(x) \geq 2^{c}$.

(ii) If for some non-discrete closed subgroup $H$ of $G$, int ${ }_{a H b} F \neq \emptyset$, then card $\operatorname{TIM}_{\mathbf{P}}(x) \geq 2^{c}$ for all $x \in$ int $_{a H b} F$.

(iii) If $R$ is a closed subgroup of $G, S \subset R$ is a symmetric set (such as the Cantor $1 / 3$ set) and $a S b \subset F$, then card $\operatorname{TIM}_{\mathbf{P}}(x) \geq 2^{c}$ for all $x \in a S b$, provided $p=2$.

For (i) see [Gr3, Theorem 4] and for (ii), (iii) see [Gr4, Theorems 6,7$]$.

THEOREM 6. Let $G$ be a locally compact group, $J \subset A_{p}$ a closed ideal with $Z(J)=F, a, b \in G, 1<p<\infty$. Assume that (i) or (ii) [or (iii)] of the above theorem holds. Then $\left(A_{p} / J\right)^{* *}\left[(A / J)^{* *}\right]$ is a non-amenable and non-semisimple Banach algebra.

PROOF. By Proposition 3, Theorem 4, and Theorem 5.

REMARKS. (a) In fact (the $w^{*}$-closed two-sided ideal) $\operatorname{TI}_{\mathbf{P}}^{0}(x) \subset \operatorname{rad} \boldsymbol{P}^{*}$ (since $\operatorname{TI}_{\mathbf{P}}^{0}(x) \square \operatorname{TI}_{\mathbf{P}}^{0}(x)=\{0\}$ ) and card $\operatorname{TI}_{\mathbf{P}}^{0}(x) \geq 2^{c}$. In cases (ii) or (iii) there are at least $c$ such ideals, by Proposition 3(c).

(b) In case (ii) $\left[(\right.$ iii) $] A_{p}(F)^{* *}\left[A(F)^{* *}\right]$ is not amenable.

(c) Theorem 6 says nothing about the amenability of the algebras $A / J$ or $A(F)$. For example, if $G$ is abelian then $A(G)$ is amenable, hence so are all the quotient algebras $A / J$ [or $A(F)$ ] for any closed ideal $J \subset A(G)$ [set $F \subset G$ ]. Yet for sets $F \subset G$ as in Theorem $6, A(F)^{* *}$ and $(A / J)^{* *}$ are not amenable.

The following is folklore.

Proposition 7. Let $J_{1} \subset J_{2}$ be closed ideals in $A_{p}$ with $F_{i}=Z\left(J_{i}\right)$; thus $F_{2} \subset F_{1}$. If $\left(A_{p} / J_{2}\right)^{* *}\left[A_{p}\left(F_{2}\right)^{* *}\right]$ is not amenable, then $\left(A_{p} / J_{1}\right)^{* *}\left[A_{p}\left(F_{1}\right)^{* *}\right]$ is not amenable.

PROOF. Let $q: A_{p} / J_{1} \rightarrow A_{p} / J_{2}$ be the canonical quotient onto map. Then $q^{* *}:\left(A_{p} / J_{1}\right)^{* *} \rightarrow\left(A_{p} / J_{2}\right)^{* *}$ is a homomorphism whose image $B$ contains $A_{p} / J_{2}$ (considered as embedded in $\left.\left(A_{p} / J_{2}\right)^{* *}\right)$. But by [Ru2, 4.14] $B$ has to be $w^{*}$-closed. 
Hence $q^{* *}$ is an onto continuous homomorphism and amenability is preserved by such ([CL]: see Lemma 1.1 in [LL]).

COROLLARY 8. (a) If $G$ is non-discrete then $A_{p}(G)^{* *}$ is not amenable and not semisimple.

(b) For any locally compact group $G, A(G)^{* *}\left[\right.$ or $\left.B(G)^{* *}\right]$ is amenable if and only if $G$ is finite.

Proof. (a) Take $H=G=F$ in Theorem 6(b). By Lau's result [La, Proposition 3.2(b)], if $A(G)^{* *}$ is amenable then $G$ is compact and by (a) it has to be discrete. Assume now that $B(G)^{* *}$ is amenable. Since $A(G)$ is a complemented ideal in $B(G)$, $A(G)^{* *}$ is a complemented ideal in $B(G)^{* *}$. Thus by [CL], $A(G)^{* *}$ is amenable; hence $G$ is finite.

Proposition 9. Let $A(G)$ be amenable. Then $A_{p}(G) / J$ is amenable for all $1<p<\infty$ and for all closed ideals $J \subset A_{p}(G)$.

ProOF. $G$ is necessarily amenable; hence, by Herz's Theorem C in [Hz2], $A_{2}(G) \subset$ $A_{p}(G)$ for all $1<p<\infty$, with contraction of norms. Thus the identity embedding $h: A_{2} \rightarrow A_{p}$ is a homomorphism such that $\|h\| \leq 1$. But $h A_{2}$ contains the functions $f * \tilde{g}$ with $f, g \in C_{c}(G)$, the linear span of which is norm dense in $A_{p}$ (see [Hz1]). Thus $h A_{2}$ is norm dense in $A_{p}$. By a theorem of Johnson ([Jo1, (5.3)]) $A_{p}=A_{p}(G)$, hence $A_{p} / J$ is an amenable Banach algebra.

REMARKS. (a) Proposition 9 improves Theorem 3.10 of [Fol].

(b) Corollary 8(b) is the dual result to Theorem 1.3 and Corollary 1.4 of [GLW].

(c) It has been proved by Gourdeau [Go] that for any Banach algebra $B$ the amenability of $B^{* *}$ implies that of $B$ (see also [GLW]). Thus $A(G)^{* *}$ amenable implies that $A(G)$ is so. It has been proved by Johnson in [Jo2] that there exist compact groups for which $A(G)$ is not amenable. If, however, $G$ is infinite and contains an abelian subgroup of finite index then $A(G)$, hence $A_{p}(G)$, is amenable (see [LLW, Corollary 4.2] and [Fo2]), yet $A(G)^{* *}$ is not amenable by Corollary 8(b).

(d) It has been proved by Brown and Moran [BM] that if $G$ is a non-compact abelian locally compact group then $B(G)$, hence $B(G)^{* *}$, is not amenable. If $G$ is compact abelian infinite then $A(G)=B(G)$ is amenable yet $A(G)^{* *}=B(G)^{* *}$ is not amenable by, say, our Corollary 8.

(e) If $G$ is abelian, every perfect compact set $F$ contains a perfect Helson set $E \subset F$ by Varopoulos [V, Ch. 4.3]. Taking $F \subset R=G$ to be the Cantor 1/3 (or any symmetric) set and $E \subset F$ a Helson set we get that $A(E)^{* *}$ is amenable while $A(F)^{* *}$ is not. 
(f) There exist continuous [smooth] curves $E$ in $R^{2}$ [in $R^{n}, n \geq 3$ ] which are Helson sets as shown by Kahane; see [Mc]. Thus if $G=R^{n}$ then $A(E)^{* *}$, and hence $A\left(E_{1}\right)^{* *}$ for all closed $E_{1} \subset E$, is an amenable Banach algebra.

(g) Let $G$ be infinite discrete and abelian. Then any infinite set $F \subset G$ contains an infinite Sidon set $E$; thus $A(E)=c_{0}(E)$ ([Ru 1, (5.7.3) (5.7.6)]). Hence $A(E)^{* *}=$ $C(X)$ (for some compact $X$ ) is amenable. Yet $A(F)^{* *}$ need not be amenable (take $F=G$ and use our Corollary $8(\mathrm{~b}))$.

Questions. (1) Characterize the closed sets $E \subset R^{n}$ for which $A(E)^{* *}$ is an amenable Banach algebra.

(2) Let $G=\mathbb{Z}$, the integers, or $G=\mathbb{Z}^{n}$. Characterize all infinite sets $F \subset G$ for which $A(F)^{* *}$ is amenable.

(3) The only examples of sets $E \subset G$ for which $A(E)^{* *}$ is amenable, given here, are Helson sets or Sidon sets. Do other such exist?

\section{References}

[BM] G. Brown and W. Moran, 'Point derivations in $M(G)$ ', Bull. London Math. Soc. 8 (1976), 57-64.

[CL] P. C. Curtis Jr. and R. J. Loy, 'The structure of amenable Banach algebras', J. London Math. Soc. 40 (1989), 89-104.

[DH] J. Duncan and S. A. R. Hosseinium, 'The second dual of a Banach algebra', Proc. Royal Soc. Edinburgh Sect. A 84 (1979), 309-325.

[Ey] P. Eymard, 'L'algèbre de Fourier d'un groupe localement compact', Bull. Soc. Math. France 92 (1964), 181-236.

[Fol] B. Forrest, 'Amenability and the structure of the algebras $A_{p}(G)$ ', Trans. Amer. Math. Soc. 343 (1994), 233-243.

[Fo2] - 'Amenability and weak amenability of the Fourier algebra', preprint.

[Go] F. Gourdeau, Amenability of Banach algebras (Ph. D. Thesis, Cambridge University, 1989).

[Gr1] E. Granirer, 'On some spaces of linear functionals on the algebras $A_{p}(G)$ for locally compact groups', Colloq. Math. 52 (1987), 119-132.

[Gr2] _ - 'On convolution operators with small support which are far from being convolution by a bounded measure', Colloq. Math. 67 (1994), 33-60.

[Gr3] _ 'Day points for quotients of the Fourier algebra $A(G)$, extreme nonergodicity of their duals and extreme non Arens regularity', Illinois J. Math. 40 (1996), 402-419.

[Gr4] _ _ ' 'On the set of topologically invariant means on an algebra of convolution operators on $L^{p}(G)^{\prime}$, Proc. Amer. Math. Soc. 124 (1996), 3399-3406.

[GLW] F. Ghahramani, R. J. Loy and G. A. Willis, 'Amenability and weak amenability of second conjugate Banach algebras', Proc. Amer. Math. Soc. 124 (1996), 1489-1497.

[GMc] C. C. Graham and O. C. McGehee, Essays in commutative harmonic analysis (Springer, New York, 1979).

[Hz1] C. Herz, 'Harmonic synthesis for subgroups', Ann. Inst. Fourier (Grenoble) 37 (1973), 91-123. 
[Hz2] - 'The theory of $p$-spaces with an application to convolution operators', Trans. Amer. Math. Soc. 154 (1971), 69-82.

[Jo1] B. E. Johnson, 'Cohomology in Banach algebras', Mem. Amer. Math. Soc. 127 (Amer. Math. Soc., Providence, 1972).

[Jo2] _ _ 'Weak amenability of group algebras', Bull. London Math. Soc. 23 (1991), 281-284.

[La] A. T.-M. Lau, 'The second conjugate algebra of the Fourier algebra of a locally compact group', Trans. Amer. Math. Soc. 267 (1981), 53-63.

[LL] A. T.-M. Lau and R. J. Loy, 'Amenability of convolution algebras', Math. Scand., to appear.

[LLW] A. T.-M. Lau, R. J. Loy and G. A. Willis, 'Amenability of Banach and $C^{*}$ algebras on locally compact groups', Studia Math. 119 (1996), 161-178.

[Mc] O. C. McGehee, 'Helson sets in $T^{n}$ ', in: Conference in Harmonic Analysis, College Park Maryland 1971, Lecture Notes in Math. 266 (Springer, Berlin, 1979) pp. 229-237.

[Ru1] W. Rudin, Fourier analysis on groups (Interscience, New York, 1960).

[Ru2] _- Functional analysis (McGraw-Hill, New York, 1973).

[V] N. Th. Varopoulos, 'Tensor algebras and harmonic analysis', Acta Math. 119 (1967), 51-112.

Department of Mathematics

University of British Columbia

Vancouver V6T 1 Z2

Canada

e-mail: granirer@math.ubc.ca 